\title{
Prediction of shear-band thickness in metallic glasses
}

\author{
M.Q. Jiang, ${ }^{\mathrm{a}}$ W.H. Wang ${ }^{\mathrm{b}}$ and L.H. Dai ${ }^{\mathrm{a}, *}$ \\ ${ }^{a}$ State Key Laboratory of Nonlinear Mechanics, Institute of Mechanics, Chinese Academy of Sciences, Beijing 100190, \\ People's Republic of China \\ ${ }^{\mathrm{b}}$ Institute of Physics, Chinese Academy of Sciences, Beijing 100190, People's Republic of China
}

Received 20 November 2008; revised 31 January 2009; accepted 20 February 2009

Available online 27 February 2009

\begin{abstract}
We derive an explicit expression for predicting the thicknesses of shear bands in metallic glasses. The model demonstrates that the shear-band thickness is mainly dominated by the activation size of the shear transformation zone (STZ) and its activation free volume concentration. The predicted thicknesses agree well with the results of measurements and simulations. The underlying physics is attributed to the local topological instability of the activated STZ. The result is of significance in understanding the origin of inhomogeneous flow in metallic glasses.
\end{abstract}

(c) 2009 Acta Materialia Inc. Published by Elsevier Ltd. All rights reserved.

Keywords: Metallic glasses; Shear band; Shear transformation zone; Free volume

The shear-banding-mode plastic flow of metallic glasses (MGs) at ambient temperature continues to fascinate and challenge scientists [1-5] because of its physical origin and practical implications. The free volume creation [6] and local heating generation [7], in which shear-band thickness is an important factor $[2,8]$, are two potential causes of shear-banding instability in MGs. Here, the shear-band thickness is the characteristic width of the strain localization normal to the shear plane, and is not involved in the ultimate failure. In general, the thickness of shear bands in MGs is restricted to a rather narrow range from several to 10 or more nanometers, regardless of chemical components and loading methods (including tension, compression, bending, indentation, rolling, etc.); this has been widely found by direct experimental observations [9-15], or by numerical simulations [16-18]. Such localization of plastic flow, far smaller than the thickness $(10-500 \mu \mathrm{m})$ of adiabatic shear bands (ASBs) in conventional alloys [19], suggests that the shear bands in MGs have a structural, rather than a thermal, origin $[1,2,8]$. Furthermore, the coupled thermomechanical analysis of shear-banding instability in MGs reveals that the onset of this instability is mainly controlled by local free volume softening [20] via discrete atomic jumps [6] or cooperative arrangement of local atomic clusters, termed the "shear transformation zone" (STZ) [21] or "flow defect" [22]. The

\footnotetext{
*Corresponding author. Tel.: +86 10 82543931; fax: +86 10 82543977; e-mail: lhdai@Inm.imech.ac.cn
}

STZ is the fundamental unit process underlying plastic deformation associated with the free-volume evolution. Although the study of thickness provides insight into the origin of shear-banding instability, the theoretical prediction of the shear-band thickness itself in MGs lags well behind that of ASBs in crystalline alloys.

Very recently, Joshi and Ramesh [23] have predicted a shear-band thickness of about $10-50 \mathrm{~nm}$, based on a rotational plastic deformation mechanism in nanocrystalline materials at grain sizes approaching the amorphous limit $(\sim 2 \mathrm{~nm})$. The lower bound of their predicted thickness agrees well with the shear-band thicknesses for many MGs [2]. This means that the flipping of STZ may be valid for the shear localization process in MGs. In addition, finite STZ sizes of about 1-2 $\mathrm{nm}$ (not reaching $2 \mathrm{~nm}$ ) have been identified by many recent works [24-28]. Interestingly, the "10-time-rule" in granular materials [29] seems to be roughly satisfied in MGs, i.e. the shear-band thickness is approximately 10 times the STZ size, implying a similar shear instability mechanism between the two materials. However, the quantitative relationship between the thickness of the shear band and the size of the STZ is still under investigation, and the underlying precise physics that dominates the shear-band thickness is not clear. In this paper, we present an explicit expression of the thickness of shear banding based on shear instability due to STZs in metallic glasses. Its underpinning nature is discussed.

Plastic deformation of MGs occurs by the cascade of STZs or flow defects $[3,21,22,30]$. Subjected to an 
external shear stress $\tau$, an STZ with activation volume $\Omega_{a}$ undergoes a characteristic shear strain $\gamma_{a}(\approx 1)$ at a rate that depends on $\tau$. The concentration of STZs $C_{\xi}$, namely the fraction of material that is available to STZ operations in a unit volume element, is statistically related to the free volume concentration $\xi$ by $C_{\xi}=\exp (-1 / \xi)[3,22]$. Thus, the STZ operations in unit volume element can be characterized by the evolution of $\xi$ as $[20,31]$ :

$\frac{\partial \xi}{\partial t}=D_{\xi} \frac{\partial^{2} \xi}{\partial y^{2}}+G(\tau, \xi)$,

where $D_{\xi}$ is the diffusion coefficient of free volume concentration [31], and the net creation rate function $G$ is the combined rate of annihilation and generation of free volume, and is taken to be dependent on the local concentration of free volume $\xi$, and the shear stress $\tau$ [6]. The explicit expression of $G$ was given first by Spaepen [6], as follows:

$$
\begin{aligned}
G(\tau, \xi)= & \frac{1}{\chi} v_{0} \exp \left[-\frac{1}{\xi}\right] \exp \left[-\frac{\Delta G^{m}}{k_{B} T}\right] \\
& \times\left\{\frac{2 k_{B} T}{\xi v^{*} S}\left[\cosh \left(\frac{\tau \Omega}{2 k_{B} T}\right)-1\right]-\frac{1}{n_{D}}\right\},
\end{aligned}
$$

where $\chi$ is a geometrical factor, $v^{*}$ is the critical volume or the effective hard-sphere size of an atom, the attempt frequency $v_{0}$ is essentially the frequency (approximately the Debye frequency) of the fundamental mode vibration along the reaction pathway [22], $\Delta G^{m}$ is the activation energy, $k_{B} T$ is the thermal energy, $S$ is the Eshelby modulus $(S=2(1+v) \mu / 3(1-v)$ where $\mu$ is the shear modulus $v$ is the Poisson's raio), $\Omega$ is the atomic volume, and $n_{D}$ is the number of diffusive jumps necessary to annihilate a free volume equal to $v^{*}$. Meanwhile, the macroscopic plastic strain rate can be written as $[3,21,22]$ :

$\dot{\gamma}^{p}=C_{\xi} \gamma_{a} H_{a}$,

where the net activation frequency $H_{a}$ of an STZ obeys a rate law of the form [3]: $H_{a}=v_{0}\left[\exp \left(-\frac{Q-\tau \Omega_{a}}{k_{B} T}\right)\right.$ $\left.-\exp \left(-\frac{Q+\tau \Omega_{a}}{k_{B} T}\right)\right]$, here $Q$ is the activation energy barrier for an STZ under an unstressed field [30]. In addition, the momentum balance, in the absence of body forces, requires that $[20,31]$ :

$\rho \frac{\partial^{2} \gamma}{\partial t^{2}}=\frac{\partial^{2} \tau}{\partial y^{2}}$

where $\rho$ is the mass density, and the total shear strain $\gamma$ can be decomposed into elastic and plastic parts, i.e. $\gamma=\gamma^{e}+\gamma^{p}$, here the elastic strain $\gamma^{e}$ obeys Hooke's law: $\gamma^{e}=\tau / \mu$. Eqs. (1)-(4) govern the inhomogeneous deformation of MGs.

Shear banding, as a physically unstable event, is investigated through a linear perturbation analysis, i.e. seeking an inhomogeneous solution with respect to small perturbations to the homogeneous solution. The homogeneous solution $\left(\tau_{h}, \gamma_{h}, \xi_{h}\right)$ satisfies $\partial \tau_{h} / \partial y=\partial \gamma_{h} /$ $\partial y=\partial \xi_{h} / \partial y=0$. For a typical $\mathrm{Zr}_{41.2} \mathrm{Ti}_{13.8} \mathrm{Cu}_{12.5-}$ $\mathrm{Ni}_{10} \mathrm{Be}_{22.5}$ (Vit 1) BMG, Figure 1 shows the stress-strain curve (black) of homogeneous deformation, along with the concentration of free volume vs. shear strain for a strain rate of $10^{-2} \mathrm{~s}^{-1}$. For small perturbations it is assumed that $(\delta \tau, \delta \gamma, \delta \xi)=\left(\tau^{*}, \gamma^{*}, \xi^{*}\right) \exp (\alpha t+\mathrm{i} k y)$, where $\left(\tau^{*}, \gamma^{*}, \xi^{*}\right)$ are small constants that characterize the initial magnitude of the perturbation, $k$ is the wavenumber, and $\alpha$ is related to the initial rate of growth. The stability of the deformation is now determined by the sign of the real part of $\alpha$ : if $\operatorname{Re}(\alpha)<0$, the shear deformation is stable; if $\operatorname{Re}(\alpha)>0$, it is unstable. The stability analysis tells immediately that the critical wavelength that may lead to runaway instability is:

$\lambda_{\text {crit }}=2 \pi\left(\frac{D_{\xi}}{G_{\xi}^{*}}\right)^{1 / 2}$.

where $G_{\xi}^{*}$ is the free volume coalescence rate $\partial G / \partial \xi$ at the critical point of instability. Perturbations with a wavelength smaller than $\lambda_{\text {crit }}$ will die out, whereas the ones with a wavelength larger than $\lambda_{\text {crit }}$ will grow. Based on the homogeneous solution (Fig. 1), we can obtain the

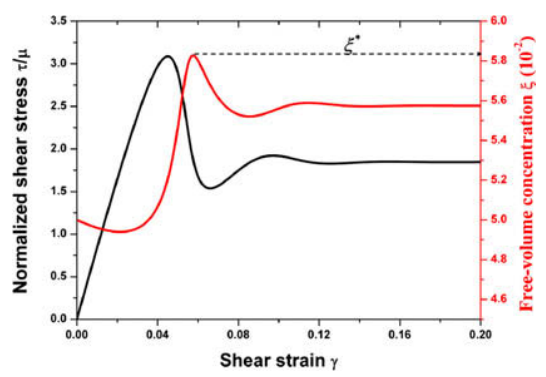

Figure 1. Dimensionless shear stress (black curve) and free volume concentration (red curve) vs. shear strain for the homogeneous deformation at a strain rate of $10^{-2} \mathrm{~s}^{-1}$; the peak value of free volume concentration is denoted by $\xi^{*}$. (For interpretation of the references to color in this figure legend, the reader is referred to the web version of this article.)
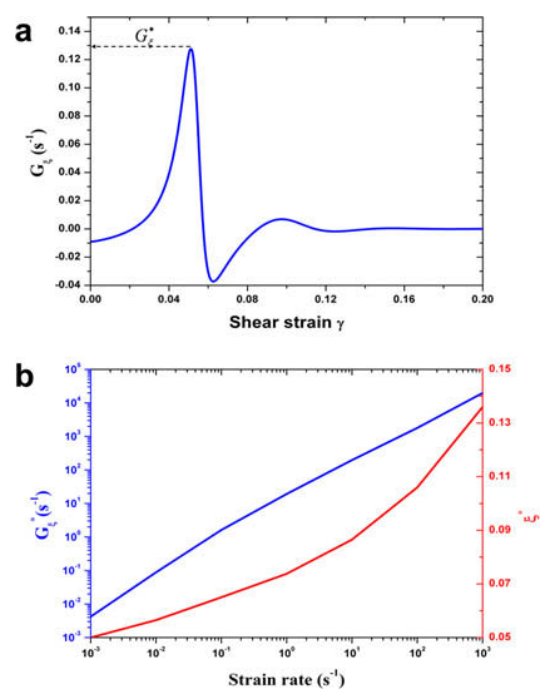

Figure 2. (a) Evolution of coalescence rate $G_{\xi}$ of free volume concentration with shear strain at a strain rate of $10^{-2} \mathrm{~s}^{-1}$; its peak value is denoted by $G_{\xi}^{*}$. (b) The effect of applied shear strain rate on $G_{\xi}^{*}$ and $\xi^{*}$. 
variation in $G_{\xi}=\partial G / \partial \xi$ with shear strain at a strain rate of $10^{-2} \mathrm{~s}^{-1}$, as shown in Figure 2a. Obviously, $G_{\xi}$ reaches the peak value $\max \left[G_{\xi}\right]$ at the critical point of instability; hence, $G_{\xi}^{*}=\max \left[G_{\xi}\right]$. Furthermore, it is noted that, as the strain rate ranges from $10^{-3}$ to $10^{3} \mathrm{~s}^{-1}, G_{\xi}^{*}$ spans about seven orders of magnitude (from $\sim 10^{-3}$ to $10^{4} \mathrm{~s}^{-1}$ ), as shown in Figure $2 \mathrm{~b}$ by the blue curve. The $G_{\xi}^{*}$ displays a highly positive rate-dependence. According to Eq. (5), the critical wavelength $\lambda_{\text {crit }}$ only depends on $G_{\xi}^{*}$ if $D_{\xi}$ remains approximately constant before instability [20,31]. That is why shearband instability is more probable at high strain rates, which is consistent with the available experimental observations [32-34].

In fact, the critical wavelength is the embryonic nuclei size of shear-banding instability, which is only valid for a short time after instability [31]. The thickness of observed shear banding often corresponds to the wavelength that grows most quickly or the dominant wavelength of the instability, $\lambda_{m}$ [19]. However, many works conclude that $\lambda_{m}$ should be of the order of $\lambda_{\text {crit }}$ [23,35-37]. Consequently, we can reasonably assume that the thickness of a mature shear band is the final dimension of the perturbation with the initial wavelength $\lambda_{\text {crit }}$, developing into the local steady-state flow in the post-instability stage. In this stage, the diffusion coefficient of the free volume concentration is expected to obey the Stokes-Einstein equation [6,38]: $D_{\xi}=\frac{k_{B} T}{6\left(v_{f} / d^{2}\right) \eta}$, where $v_{f}$ is the absolute free volume (approximately the atomic volume), $d$ is the diffusion distance of free volume, and the shear viscosity can be derived: $\eta=\tau_{y} / \dot{\gamma}^{p}$ with the flow stress $\tau_{y}$. As mentioned previously, the STZ is a local rearrangement of atoms around a high free volume site under shear stress. The free volume site, as internal structural defects in $\mathrm{MG}$, is analogous to a dislocation in a crystal. A dislocation can move a long distance only if its neighboring atoms make a little move (usually a distance of less than a lattice periodic). By the same token, it is plausible that the free volume site can move or diffuse a distance of an equivalent diameter or size of STZ when a STZ disappears due to itself-induced free volume increase. Therefore, $d$ is assumed to be roughly equal to the STZ size. In addition, at the stress level $\tau_{y}$, the free volume coalescence rate can reduce to [39] $G_{\xi} \approx a \dot{\gamma}^{p}$, where $a=\left(k_{B} T / S\right)\left(1 / \gamma_{a} v_{f} \xi\right)$. Then, we obtain the explicit expression of the shear-band thickness:

$\left.W \approx \lambda_{\text {crit }}\right|_{\text {steady state }}=\frac{2 \pi}{3} d\left(\xi^{*} \cdot \frac{1}{\gamma_{C}} \cdot \frac{1+v}{1-v}\right)^{1 / 2}$

where $\xi^{*}$ is the activation free volume concentration due to STZ-induced shear instability (see below). The average shear yield strain $\gamma_{C}=\tau_{y} / \mu$ is almost a universal constant of about 0.0267 for MGs [30]. This equation indicates that the correlation between the shear-band thickness and the STZ size does not follow a simple linear relation, and the influence of other parameters such as $\xi^{*}, \gamma_{C}$ and $v$ should be included.

It is noted that among the parameters governing the thickness of shear bands, both $d$ and $\xi^{*}$ are often difficult to determine precisely. Very recently, based on the John-
son-Sawmer cooperative shearing model [30], Pan et al. [28] have developed a rate-jump nanoindentation method to characterize the STZs size of MGs. They found that the equivalent sizes of STZ for a variety of MGs are of $1.3-1.9 \mathrm{~nm}$. This dimension agrees well with the range of STZ sizes predicted by many previous works [24-27]. In the following calculation, the range of $1-2 \mathrm{~nm}$ of STZ sizes $d$ and the average value of $1.5 \mathrm{~nm}$ are adopted. Let us now turn to discussion of the possible range of the activation free volume concentration $\xi^{*}$. Here, we assume that the homogenous steady state in which the amount of free volume created is equal to the amount of free volume annihilated can be achieved in a mature shear band $[21,40]$, because the shear-band thickness is very thin $(\sim 10 \mathrm{~nm})$. Therefore, $\xi^{*}$ in Eq. (6) is approximately considered as the $\xi$ value in the steady state during homogeneous deformation. As shown in Figure 1 (red curve), the $\xi$ in the steady state is very close to $\operatorname{Max}[\xi]$. Thus, we reasonably choose $\xi^{*}=\operatorname{Max}[\xi]$. The effect of strain rate on $\xi^{*}$ is shown in Figure $2 b$ by the red curve. Compared to $G_{\xi}^{*}$, the $\xi^{*}$ exhibits a strain-rate-insusceptible behavior and ranges from 0.050 to 0.135 with increasing strain rate from $10^{-3}$ to $10^{3} \mathrm{~s}^{-1}$. According to Eq. (6), $\xi^{*}$ is closely related to the shear-band thickness; this might be the main reason that the shear-band thickness is not sensitive to strain rate. Considering the broad MG systems from ideally brittle $\mathrm{Mg}$-based to typical tough $\mathrm{Zr}$-based, we reasonably choose the fiducial interval [0.001, 0.200] of $\xi^{*}$. In addition, MGs have Poisson's ratios $v$ of $\sim 0.28$ $0.42[41,42]$. We first examine the influences of $v$ and $\xi^{*}$ on the thickness $W$ for fixed $d=1.5 \mathrm{~nm}$, as shown in Figure 3a. The predicted shear-band thicknesses of several to ten or more nanometers exhibit a fair agreement with the available experimental observations and simulations [9-18]. $W$ is insensitive to $v$ but increases significantly with increasing $\xi^{*}$, indicating $\xi^{*}$ is a master factor. Then, we take a typical value $(0.36)$ of $v$, and further explore the effect of the STZ sizes $d$ on the thickness, as shown in Figure $3 \mathrm{~b}$. We find that $W$ is also strongly dependent on $d$. Thus, one can conclude that the shear-band thickness in MGs is mainly governed by the STZ-activated free volume concentration incurring instability, and the size of the STZ.

Next, we discuss the physical picture behind Eq. (6). Since the fundamental unit process underlying plastic flow is a STZ, the activation free volume facilitating local shear instability is actually the threshold of free volume
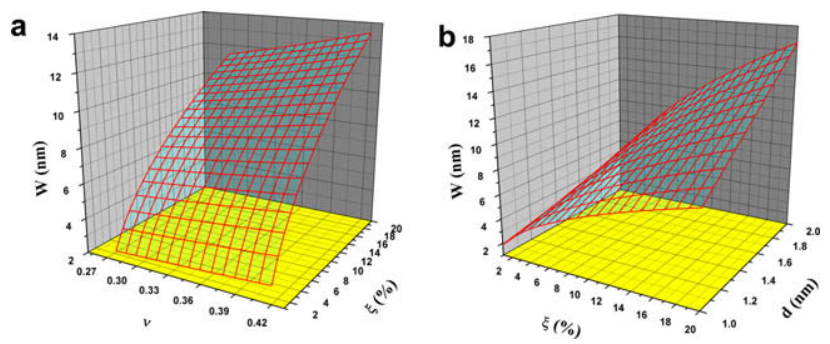

Figure 3. Prediction of shear-band thickness $W$. (a) Dependence of Poisson's ratio $v$ and activation free volume concentration $\xi^{*}$ on $W$ for fixed STZ size $d=1.5 \mathrm{~nm}$. (b) Dependence of $\xi^{*}$ and $d$ on $W$ for fixed $v=0.36$. 
in activated STZs, beyond which the STZs become topologically unstable. At the atomic cluster level, the free volume threshold must be a probability (usually Gaussian) distribution [6,21]. Egami's theory of topological fluctuations in the bonding network has predicted that if the average local transformation volume strain is larger than about $10 \%$, the local atomic cluster site will become topologically unstable and be liquid-like [43]. This average local volume strain can be analogous to the mathematical expectation of the probability distribution of local STZ-activated free volume concentration in plastic flow. In addition, the recent work of Liu et al. [44] has also revealed that there is a dividing line of free volume concentration of about 0.10 between a densely packed structure with coordination number $(\mathrm{CN})$ of $>10$ and loose one with $\mathrm{CN}<10$. Therefore, we reasonably choose $\xi^{*} \approx 0.10, d=1.5 \mathrm{~nm}$ and $v=0.36$, and the calculated shear-banding thickness $W \approx 9 \mathrm{~nm}$ according to Eq. (6). This value agrees well with the characteristic thickness $(\sim 10 \mathrm{~nm})$ of shear bands in various MGs [2], demonstrating the intrinsic universality of local topological stability of STZs in glassy structures undergoing inhomogeneous plastic flow. In crystalline alloys, the thickness of ASBs is controlled by a balance of between plastic work and heat diffusion; its theoretical prediction has been successfully established [19]. However, the shear-band thickness in MGs is underpinned by a balance of free volume creation-diffusion via superposition of STZs. Thus, the local topological instability of STZs, tying up with their activation size and free volume, determines the thickness of shear bands in MGs, as predicted by explicit expression (6) in the present work.

In summary, this study provides a physical understanding of the shear-band thickness in MGs based on STZ-induced shear instability analysis. In particular, an explicit expression of the thickness of shear bands, which has not previously been available, is derived, and can explain why the shear-band thicknesses of various MGs narrow down to around the order of $10 \mathrm{~nm}$. The good agreement between theoretical predictions and experimental observations provides compelling evidence that the shear instability of MGs originates from local STZs activated by shear stresses. The findings have implications for understanding of the atomic-scale flow mechanisms of MGs. In addition, combining our prediction model (6) with Pan et al.'s experimental method of characterizing STZs may provide a rough, but useful, approach to estimate the free volume concentration within shear bands in MGs.

Financial support for this work came from the Natural Science Foundation of China (Grants Nos. 10725211, 10721202 and 50731008), the National Basic Research Program of China (Grant No. 2009CB724401) and the Knowledge Innovation Project \& Key Project of Chinese Academy of Sciences (Nos. KJCX2-YW-M04 and KJCX-SW-L08).

[1] J.J. Lewandowski, A.L. Greer, Nat. Mater. 5 (2006) 16. [2] Y. Zhang, A.L. Greer, Appl. Phys. Lett. 89 (2006) 071907.
[3] C.A. Schuh, T.C. Hufnagel, U. Ramamurty, Acta Mater. 55 (2007) 4067.

[4] Y.H. Liu, G. Wang, R.J. Wang, D.Q. Zhao, M.X. Pan, W.H. Wang, Science 315 (2007) 1385.

[5] D.C. Hofmann, J. Suh, A. Wiest, G. Duan, M. Lind, M.D. Demetriou, W.L. Johnson, Nature 451 (2008) 1085.

[6] F. Spaepen, Acta Metall. 25 (1977) 407.

[7] H.J. Leamy, H.S. Chen, T.T. Wang, Metall. Trans. 3 (1972) 699.

[8] F. Spaepen, Nat. Mater. 5 (2006) 7.

[9] T. Masumoto, R. Maddin, Acta Metall. 19 (1971) 725.

[10] P.E. Donovan, W.M. Stobbs, Acta Metall. 29 (1981) 1419.

[11] E. Pekarskaya, C.P. Kim, W.L. Johnson, J. Mater. Res. 16 (2001) 2513.

[12] J. Li, F. Spaepen, T.C. Hufnagel, Phil. Mag. 82 (2002) 2623.

[13] W.H. Jiang, M. Atzmon, Scripta Mater. 54 (2006) 333.

[14] G. Rizza, A. Dunlop, M. Kopcewicz, Nucl. Instrum. Methods Phys. Res. B 245 (2006) 130.

[15] Q.P. Cao, J.F. Li, Y.H. Zhou, J.Z. Jiang, Scripta Mater. 59 (2008) 673.

[16] Y. Shi, M.L. Falk, Phys. Rev. Lett. 95 (2005) 095502.

[17] N.P. Bailey, J. Schiøtz, K.W. Jacobsen, Phys. Rev. B 73 (2006) 064108.

[18] R. Matsumoto, N. Miyazaki, Scripta Mater. 59 (2008) 107.

[19] Y.L. Bai, B. DoddAdiabatic Shear Localization: Occurrence, Theories and Applications, Pergamon Press, Oxford, 1992.

[20] L.H. Dai, M. Yan, L.F. Liu, Y.L. Bai, Appl. Phys. Lett. 87 (2005) 141916.

[21] A.S. Argon, Acta Metall. 27 (1979) 47.

[22] F. Spaepen, Physics of Defects, North-Holland, Amsterdam, 1981.

[23] S.P. Joshi, K.T. Ramesh, Phys. Rev. Lett. 101 (2008) 025501.

[24] D.B. Miracle, Nat. Mater. 3 (2004) 697.

[25] S.G. Mayr, Phys. Rev. Lett. 97 (2006) 195501.

[26] M. Zink, K. Samwer, W.L. Johnson, S.G. Mayr, Phys. Rev. B 73 (2006) 172203.

[27] H.W. Sheng, W.K. Luo, F.M. Alamgir, J.M. Bai, E. Ma, Nature 439 (2006) 419.

[28] D. Pan, A. Inoue, T. Sakural, M.W. Chen, PNAS 105 (2008) 14769.

[29] B. Francois, F. Lacombe, H.J. Herrmann, Phys. Rev. E 65 (2002) 031311.

[30] W.L. Johnson, K. Samwer, Phys. Rev. Lett. 95 (2005) 195501.

[31] R. Huang, Z. Suo, J.H. Prvost, W.D. Nix, J. Mech. Phys. Solids 50 (2002) 1011.

[32] C.A. Schuh, T.G. Nieh, Acta Mater. 51 (2003) 5187.

[33] L.F. Liu, L.H. Dai, Y.L. Bai, B.C. Wei, J. Non-Cryst. Solids 351 (2005) 3259.

[34] L.F. Liu, L.H. Dai, Y.L. Bai, B.C. Wei, J. Eckert. J. Mater. Res. 21 (2006) 153.

[35] W.W. Mullins, R.F. Sekerka, J. Appl. Phys. 35 (1964) 444.

[36] F. Spaepen, Acta. Metall. 23 (1975) 615.

[37] A.S. Argon, M. Salama, Mater. Sci. Eng. 23 (1976) 219.

[38] M.H. Cohen, D. Turnbull, J. Chem. Phys. 31 (1959) 1164.

[39] M. Heggen, F. Spaepen, M. Feuerbacher, J. Appl. Phys. 97 (2005) 033506.

[40] P.S. Steif, F. Spaepen, J.W. Hutchinson, Acta Metall. 30 (1982) 447

[41] W.H. Wang, J. Appl. Phys. 99 (2006) 093506.

[42] M.Q. Jiang, L.H. Dai, Phys. Rev. B 76 (2007) 054204.

[43] T. Egami, S.J. Poon, Z. Zhang, V. Keppens, Phys. Rev. B 76 (2007) 024203.

[44] X.J. Liu, G.L. Chen, X. Hui, T. Liu, Z.P. Lu, Appl. Phys. Lett. 93 (2008) 011911. 